\title{
La huerta escolar con el uso de las TIC, la excusa perfecta para resolver problemas matemáticos
}

\author{
The School Garden with the use of ICT: the perfect excuse to solve \\ mathematical problems
}

\section{A horta escolar com o uso das TIC, a desculpa perfeita para resolver problemas matemáticos}

LIGIA SuÁREZ RodrígueZ

\begin{abstract}
* PhD. en educación. Licenciada en Ciencias Religiosas y Ética, especialista en administración de la informática educativa, magíster en gestión de la tecnología educativa. Docente de la Secretaría de Educación de Aratoca, Santander, Colombia. ligiasuaro@gmail.com https://orcid.org/0000-0001-5164-5640
\end{abstract}

\section{OPEN ACCESS $\boldsymbol{\rho}$ \\ DOI: http://dx.doi.org/10.18634/sophiaj. 17v.2i.959}

Información del artículo

Recibido: octubre 28 de 2019

Revisado: enero 30 de 2020

Aceptado: febrero 15 de 2021

Publicado: Abril 20 de 2021

Palabras clave: huerta escolar, matemáticas, educación, tics, enseñanza.

Keywords: school garden, math, education, tics, teaching.

Palavras-chave: horta escolar, matemática, educação, tiques, ensino.

Cómo citar: /how cite:

Suárez, L. (2021) La huerta escolar con el uso de las TIC, la excusa perfecta para resolver problemas matemáticos. Sophia 17 (2), e959

Sophia-Educación, volumen 17 número 2. enero/junio 2021. Versión españo
REsumen

El objetivo de esta investigación se fundamenta en resolver problemas de matemáticas desde la huerta escolar y el uso de las TIC con los estudiantes de los grados tercero, cuarto y quinto de la Institución Educativa Clavellinas. La metodología cualitativa de tipo acción participativa aplicada en esta investigación favorece una interacción activa de los agentes y sujetos participantes, la muestra se hace de manera intencional o conveniencia y los instrumentos de recolección de datos utilizados aplicados fueron entrevistas, encuestas, observaciones participantes, y secuencia didáctica. Con la Investigación aplicada se demuestra que el articular la huerta escolar como estrategia pedagógica permite avances significativos en el desarrollo de la resolución de problemas de los cinco pensamientos: numérico, espacial, métrico, aleatorio y variacional también se reconoce la importancia de adquirir buenos hábitos alimenticios con el consumo de verduras en la dieta alimentaria. La investigación da validez a la estrategia pedagógica de articular la huerta escolar al área de matemáticas pues los estudiantes adquieren habilidades y destrezas para formular y resolver problemas matemáticos de manera creativa y competente en contexto. 
Copyright 2021 Universidad La Gran Colombia

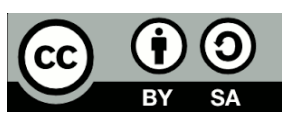

Conflicto de interés:

Los autores declaran no tener ningún conflicto de interés.

Correspondencia de autor:

Iligiasuaro@gmail.com

\section{AbSTRACT}

The objective of this research is based on solving mathematical problems from the School Garden and the use of ICT with students from third, fourth and fifth grades of the Clavellinas Educational Institution. The qualitative methodology of participatory action research type applied in this study favors an active interaction of the participating agents and subjects. The sample was selected intentionally or by convenience and the data collection instruments used were interviews, surveys, participant observations, and didactic sequence. The applied research demonstrates that the articulation of the school garden as a pedagogical strategy allows significant advances in the development of problem solving in the five areas of thinking: numerical, spatial, metric, random and variational, and also recognizes the importance of acquiring good eating habits with the consumption of vegetables in the diet. The research validates the pedagogical strategy of articulating the school garden to the area of mathematics, since students acquire skills and abilities to formulate and solve mathematical problems in a creative and competent manner in context.

RESUMO

O objetivo desta pesquisa baseia-se na resolução de problemas matemáticos da Horta Escolar e na utilização das TIC com alunos do terceiro, quarto e quinto anos da Instituição Educacional Clavellinas. A metodologia qualitativa do tipo ação participativa aplicada nesta pesquisa favorece uma interação ativa dos agentes e sujeitos participantes, a amostra é feita intencionalmente ou convenientemente e os instrumentos de coleta de dados utilizados foram entrevistas, inquéritos, observações participantes e sequência didática. Com a investigação aplicada mostra-se que articular a horta escolar como estratégia pedagógica permite avanços significativos no desenvolvimento da resolução de problemas dos cinco pensamentos: numérico, espacial, métrico, aleatório e variacional, sendo também reconhecida a importância da aquisição de bons hábitos alimentares como o consumo de vegetais na dieta alimentar. A pesquisa valida a estratégia pedagógica de articular a horta escolar à área da matemática à medida que os alunos adquirem competências e aptidões para formular e resolver problemas matemáticos de forma criativa e competente em contexto. 


\section{Introducción}

Ser docente de una zona rural exige al maestro salir de su zona de confort y enfrentarse a contextos con situaciones particulares que afectan los procesos de aprendizaje, sin embargo, la meta de formar estudiantes competentes, que adquieran un "saber hacer" para resolver las múltiples problemáticas a las que se enfrentan es constante o indistinta de la zona. Por eso, la investigación presentada en este artículo les permite a los maestros ilustrarse y aplicar en sus aulas de clase una estrategia pedagógica que se vale de un espacio de aprendizaje práctico y autentico como la huerta escolar para lograr que sus estudiantes se enamoren del área de matemáticas al estudiarla de una manera creativa, dinámica y diferente, por ende, el aplicar esta estrategia en su quehacer pedagógico, los maestros verán cómo incide notablemente en la capacidad del estudiante para analizar y comprender un problema matemático, pero a la vez diseñar y plantear soluciones.

La huerta escolar como estrategia pedagógica se ha planteado en diversos escenarios a nivel nacional e internacional. Estas experiencias se han desarrollado de manera extracurricular con el propósito de cosechar alimentos para llevar a casa. Otras se han llevado a cabo para que los estudiantes aprendan las técnicas de siembra y cosecha y el uso de las herramientas. Algunas experiencias han logrado incorporar la huerta en la escuela y con ella han logrado fomentar aprendizajes relacionados con las ciencias naturales, competencias ciudadanas y competencias científicas. No obstante, son escasas las investigaciones y experiencias que muestran el desarrollo de competencias propias de la matemática desde la huerta escolar. Este cuerpo teórico enriquece y da sustento a la investigación propuesta.

Esta investigación permite analizar el avance de los estudiantes en los pensamientos numérico, geométrico y variacional, en tanto que, logran comprender la parte global del problema matemático y su representación gracias a la comprensión del vocabulario que se utiliza. En cuanto al análisis del problema, adquieren habilidades para realizar una ordenación lógica de las partes del problema: incógnita, reconocen la pregunta que deben contestar, organizan los datos. Con respecto al razonamiento del problema, deciden con mayor facilidad las operaciones para obtener el resultado, en síntesis, adquieren competencias matemáticas de manera creativa donde el entorno, en este caso, la huerta escolar se convierte en otra aula de clase.

Esta propuesta se constituye en un piloto para aplicar en todo el municipio de Aratoca con el fin de evaluar su validez como estrategia pedagógica y buscar su aplicación a nivel provincial y luego departamental.

Este trabajo de investigación se enfoca en el desarrollo de competencias matemáticas, desde el fortalecimiento de los pensamientos numérico, geométrico y variacional, así como de las competencias o habilidades investigativas (Reyes, 2013): cognitivas, personales, metodológicas, colaborativas, informáticas y epistémicas en los niños de grado cuarto y quinto de primaria de la Institución Educativa Clavellinas del Municipio de Aratoca, Santander, Colombia. Como escenario práctico de aprendizaje se desarrollará una huerta escolar con la participación activa de los padres de familia ya que en sus hogares cada estudiante practica lo aprendido en su huerta familiar.

Al realizar la investigación se pretende obtener en un primer momento información acerca de las fortalezas y debilidades que presentan los estudiantes en el desarrollo de las competencias matemáticas. Con base en este diagnóstico, se plantea el diseño de una estrategia pedagógica basada en el manejo de la huerta escolar para propiciar el desarrollo de competencias matemáticas, especialmente en la resolución de problemas y la investigación escolar en los estudiantes de grados tercero, cuarto y quinto. Finalmente, se espera lograr información sobre la pertinencia y trascendencia de la estrategia diseñada a partir de los resultados obtenidos al aplicarla sistemáticamente en el aula.

\section{Metodología}

Se utilizó la metodología cualitativa de tipo acción participativa, ya que desde la huerta escolar, los estudiantes, e incluso padres de familia son participantes activos en la problemática investigada con el fin de que los niños y niñas desarrollen competencias o habilidades matemáticas para resolver problemas en los diferentes pensamientos del área y mejorar la calidad de nutrición en la comunidad educativa, donde la transversalidad de las áreas del conocimientos juegan un papel fundamental desde la huerta escolar y casera implementados y apoyados por el uso de las TIC en la investigación propuesta.

La investigación se realiza en la Institución Educativa Clavellinas, ubicada en el municipio de Aratoca, en la vereda Clavellinas con nueve sedes con un total de 450 estudiantes (237 de pos primaria -media rural y 213 de primaria). 
La población son los estudiantes de los grados tercero, cuarto y quinto: 69 estudiantes entre todas las sedes, se eligen estos grados, ya que las falencias a nivel académico especialmente en el pensamiento de resolución de problemas se perciben de manera más notoria en estos grados al evidenciarse en los resultados de las pruebas externas (pruebas saber), que se realizan en los grados tercero y quinto por parte del Icfes.

La muestra está conformada por los niños y niñas de los grados terceros, cuarto y quinto de primaria de las sedes Toma de San Carlos y San Ignacio, son niños y niñas en edad de 8 a 13 años, en total son 15 niños y 24 niñas para un total de 39 estudiantes, pues son las sedes más cercanas para realizar el trabajo y son las de mayor número de estudiantes que permiten un margen de error menor.

Antes de implementar la estrategia pedagógica se elaboró un DOFA: dificultades, oportunidades, fortalezas y amenazas a nivel de la gestión administrativa que permitió verificar recursos y gestión académica para verificar hipótesis y problemática planteada.

\section{Estrategia pedagógica aplicada}

Se realizó el diseño de la estrategia pedagógica a partir del diagnóstico realizado (DOFA) y de acuerdo al plan de estudios establecido en la malla curricular del Proyecto Educativo Institucional, de esta manera, los objetivos que se formulan en esta son:

- Reconocer, analizar y representar relaciones y en particular relaciones de orden y equivalencia.

- Identificar el conjunto de los números naturales y en él, efectuar las operaciones y reconocer las relaciones que correspondan a las situaciones aditivas, multiplicativa y potenciativa.

- Ejecutar habilidades de reconocimiento lógico, crítico y objetivos.

- Distinguir, ante problemas concretos, aquellos que plantean un caso de situación aditiva, multiplicativa o potenciativa y darles solución.

Teniendo en cuenta los desempeños anteriores, se plantean las estrategias pedagógicas en TIC a implementar, de acuerdo a las competencias previstas en contexto con la huerta escolar y resolución de problemas matemáticos según los ejes temáticos del área.

Se trabajan los siguientes contenidos temáticos:

- Analizando nuestros pre saberes.

- Conociendo e implementado la huerta escolar.

- Propiedades de las verduras y beneficios en el ser humano.

- Midiendo nuestra huerta escolar.

- Comprando desde la huerta escolar.

- Interpretando gráficas desde la huerta escolar

- Elaborando recetas desde la huerta escolar.

- Socializando lo aprendido en la huerta escolar.

Se plantean actividades desde el software hot potatoes, donde se pueden crear diferentes tipos de ejercicios de manera sencilla, se puede aplicar con uso en línea o desde cualquier computador off line.

Los estudiantes después de realizar sus actividades los evidencian en Cuadernia que es una herramienta muy fácil donde el software permite insertar videos, fotos, blog, tareas ejecutadas en Word (tablas, bitácoras, etc.), sin necesidad de tener internet.

Para realizar las actividades se trabajó en jornadas completas de un día de clase “día de correlación”, ya que se tranversalizan las áreas de matemáticas, lenguaje, ciencias naturales, competencias ciudadanas y tecnología e informática, durante los periodos 1 y 2 del año lectivo escolar. 
Los estudiantes crearon nuevo conocimiento pues ellos liderados por el docente elaboraron sus actividades lo que les permitió participar de manera activa en la investigación planteada.

Este papel de los estudiantes debe pasar de ser 'consumidores' de conocimiento a creadores e innovadores. En este sentido, el alumno debe convertirse en el centro de su propio aprendizaje y experimentar, crear, equivocarse y obtener resultados para mejorar su proceso de aprendizaje. 'Learning by Doing' o ‘Aprender Haciendo' es una metodología basada en la adquisición de habilidades y competencias mediante procesos de acción. Según Borgnakke (2004), podemos cambiar y modificar el aprendizaje, pero no podemos evitar 'el hacer'. (Sánchez, 2016)

En este proyecto los estudiantes pasaron de ser unos simples espectadores a formar parte fundamental del proceso de investigación; ellos fueron constructores de nuevos conocimientos a partir de la estrategia planteada, desde el semillero de investigación: Niños Investigadores del Campo asumen su responsabilidad y liderazgo en la participación de este, de esta manera resuelven problemas matemáticos desde su planteamiento hasta su solución.

El maestro como mediador es el responsable de introducir a las estudiantes en el mundo de los científicos, buscando que esta llegue a la comprensión del mundo y del lenguaje científico. La concepción que tiene el maestro de la ciencia ligada a la pedagogía genera campos de saber, esto conlleva un cambio en la idea del maestro y su rol. (Duque,2016)

Para que el proyecto tenga más validez a nivel de la huerta escolar, los padres de familia se integran, ya que en cada hogar deben realizar una huerta casera con las mismas características de las piloto que están establecidas en cada sede participante, de esta manera para los niños y niñas será más fácil poder realizar las actividades del área de matemáticas y naturales como son observar, analizar, profundizar y realizar un seguimiento a los procesos que se van observando y luego en la institución plasmarlas en los recursos tecnológicos que se utilicen sea Cuadernia, archivos de Word donde llevan sus bitácoras de aprendizaje y blog del proyecto planteado, pero sobre todo van a tener el apoyo de sus padres en su proceso de formación y se lograran mejores resultados de lo investigado.

\section{Resultados}

Después de aplicar la estrategia pedagógica hubo una gran satisfacción, el tipo de investigación acción participativa les permitió a los estudiantes, padres de familia y docentes apropiarse del proyecto dando respuesta a la problemática e hipótesis.

El análisis DOFA en el desarrollo de la competencia matemática logró cumplir el objetivo de identificar las fortalezas y debilidades de los estudiantes, para esta actividad se tuvieron en cuenta: las planillas de calificaciones del área de matemáticas donde se evalúan los desempeños de cada período, el resultado de martes de prueba aplicados en la institución, los resultados de pruebas externas de los tres últimos años, y la estructura a nivel informático, ya que esto incide en la calidad educativa.

A continuación, se presenta el DOFA al cual se llegó después de analizar cada aspecto desde dos gestiones de proyecto educativo Institucional:

\section{Gestión administrativa}

\section{Dificultades}

No se cuenta con los recursos necesarios para invertir en los requerimientos tecnológicos.

El acceso internet solo se tiene en algunas sedes.

El poco número de computadores en cada sede dificulta el proceso de enseñanza aprendizaje.

\section{Oportunidades}

La gestión por parte de las directivas para conseguir recursos para los requerimientos tecnológicos.

Aplicar software gratuito de educación que no requieran el uso de internet: Cuadernia, hot potatoes. 
Aumento de la dotación según solicitud hecha a la secretaria de las TIC.

\section{Fortalezas}

La disposición por parte de los entes gubernamentales.

La disposición de las docentes y conocimiento de aplicación de este software.

La actitud de los niños de trabajar en grupo para aprender.

\section{Amenazas}

El hurto de los computadores que ya se ha presentado en nuestras sedes por eso tenemos tan pocos.

\section{Gestión académica}

\section{Dificultades}

Según las pruebas externas aplicadas a los estudiantes son débiles en el componente Numérico-variacional.

Débil en el componente Geométrico-métrico, representación y modelación.

Con respecto a las evaluaciones que se realizan en el aula de clase los estudiantes presentan desempeño bajo en un gran porcentaje en la resolución de problemas es decir cuando se les coloca ejercicios donde aplican las operaciones básicas.

\section{Oportunidades}

El cambio de metodología de clase haciendo uso de las TIC con el uso del Cuadernia que consiste un libro digital.

Aplicar una estrategia pedagógica con el uso del software gratuito Cuadernia y hot potatoes.

Talleres de matemáticas desde la huerta escolar.

Uso de computadores, aunque pocos en la aplicación de los temas vistos.

\section{Fortalezas}

Saben las operaciones básicas con su proceso adecuado.

Fuertes en el pensamiento aleatorio.

\section{Amenazas}

Que no se observe un mejoramiento académico aún con el cambio de metodología tradicional.

Que continúen así y no se presente mejoramiento en las pruebas externas.

En el segundo objetivo: diseñar y aplicar estrategias pedagógicas para la investigación escolar en el manejo adecuado de la huerta escolar y la resolución de problemas matemáticos utilizando software libre permitió ver los siguientes resultados desde los agentes participantes:

\section{Padres de familia}

- Los padres han asumido la responsabilidad de construir en sus hogares la huerta lo que les ha permitido en familia fortalecer sus lazos al apoyar a sus hijos con sus tareas desde la huerta escolar.

- A través de la huerta han descubierto que consumir verduras orgánicas es decir sin químicos es más sano y mejora su calidad de vida.

- Los padres han comprobado que al tener una huerta casera se ahorra dinero pues no tienen que comprarlo, sino que se toma directamente de su hogar, beneficiándose de esta manera el bolsillo familiar. 
- Reconocen que es importante que sus hijos utilicen herramientas tecnológicas que al principio como lo observamos en la recolección de datos eran reacios al uso de estas, incluso han solicitado que a través de la escuela de padres se les ofrezcan unos talleres de capacitación sobre uso del computador.

\section{Estudiantes}

Otro participe fundamental son los estudiantes que a través del semillero de investigación" Niños investigadores del Campo" (NIDECAM), participan de manera activa en el proyecto de investigación planteado." El aprendizaje significativo, según Ausubel es cuando el estudiante toma nuevos conocimientos y los relaciona o integra con lo que ya tiene puede ser de su entorno familiar o adquiridos en su sitio de enseñanza los reajusta y reconstruye un nuevo conocimiento" (Suárez, 2015) y se pudo evidenciar en lo siguiente

- Concientización de una alimentación sana a través de las verduras ya que los niños al hacer la encuesta solo comían en su mayoría harinas y azucares.

- Correlación o transversalización con otras áreas del conocimiento en este caso las áreas de ciencias naturales, matemáticas, educación física, sociales (competencias ciudadanas) e informática y tecnología que le permiten al estudiante adquirir un conocimiento en forma integral.

- El uso del software gratuito donde se pueden aplicar ejercicios de resolución de problemas hace que la clase sea más agradable lo que fomenta en el estudiante amor e interés por las áreas del conocimiento.

- Es importante resaltar el cambio de actitud frente al área de matemáticas, les fascina el día de correlación, trabajan con gusto y mucho interés, lo cual ha permitido un afianzamiento del aprendizaje con mayor facilidad.

- Al realizar las evaluaciones de este periodo especialmente en cuanto a resolución de problemas se ha observado una mejoría, incluso en la última prueba de simulacro se observa que se pasó de rendimiento bajo a medio, y varios satisfactorios o avanzados.

- Con el proyecto se sigue fortaleciendo el valor de la investigación de campo, los niños ya propusieron una nueva pregunta problémica para trabajar el próximo año con otra área del conocimiento: español ¿Aprendemos competencias lectoras en la huerta escolar?

\section{Institución educativa}

La estrategia pedagógica aplicada permitió que la Institución mejorase en una formación de calidad; la investigadora en educación Isabel Segovia sustenta y le da validez cuando dice que es necesario:

Desarrollar en los niños y jóvenes habilidades, conocimientos y valores que les permitan comprender, transformar e interactuar con el mundo en el que viven. Esto implica pasar de un aprendizaje de contenidos y de una formación memorística y enciclopédica, a una educación pertinente y conectada con el país y el mundo. Igualmente, concebir la educación como un proceso que no se agota en el sistema educativo, sino que se desarrolla de manera permanente en interacción con el mundo.(Segovia,2010).

\section{Discusión de resultados}

Desde sus orígenes uno de las actividades fundamentales de la Escuela Nueva es la implementación de las huertas escolares donde el niño construye nuevos conocimientos a través del aprendizaje significativo y constructivismo, y en el caso del área de matemáticas aprende a resolver problemas en contexto:

El conocimiento será efectivo en la medida en que repose en el testimonio de la experiencia; la escuela debe, por tanto, crear las condiciones para facilitar la manipulación y experimentación por parte de los alumnos. El niño pasa a ser, así, el elemento fundamental de los procesos educativos, y tanto los programas como los métodos tendrán que partir de sus necesidades, motivaciones e intereses. (Zubiria, 2006).

El utilizar como trabajo de campo o investigación la huerta escolar en la escuela, facilita el desarrollo de las competencias matemáticas de una manera más práctica y agradable para los estudiantes al aplicar sus conocimientos en actividades cotidianas esto permite dar respuesta al Ministerio de Educación en Colombia, cuando expresa lo siguiente: 
Las competencias matemáticas no se alcanzan por generación espontánea, sino que requieren de ambientes de aprendizaje enriquecidos por situaciones problema significativas y comprensivas, que posibiliten avanzar a niveles de competencia más y más complejos. (MEN, 2004)

Además, precisa en los lineamientos y estándares en el área de matemáticas los tres aspectos fundamentales para desarrollar: Planteamiento y resolución de problemas. Razonamiento matemático (formulación, argumentación, demostración) y comunicación matemática. Consolidación de la manera de pensar (coherente, clara, precisa).

La huerta escolar es el ambiente de enseñanza aprendizaje donde el maestro en conjunto con sus estudiantes realiza proyectos relacionados con cultivos de hierbas aromáticas y hortalizas, que les permite a estos las habilidades de observación, exploración e investigación hacia nuevos conocimientos.

Los postulados constructivistas son aplicables a cualquier área del saber y las matemáticas es una de ellas.

Todo conocimiento es construido. El conocimiento matemático es construido, al menos en parte, a través de un proceso de abstracción reflexiva. Existen estructuras cognitivas que se activan en los procesos de construcción. Las estructuras cognitivas están en desarrollo continuo y la actividad con propósito induce la transformación en las estructuras existentes. Kilpatrick, (Gómez y Rico, 1995)

Las huertas escolares también permiten ser un instrumento innovador de sustentabilidad donde de manera transversal se ofrece el conocimiento, fortalece el proceso de enseñanza aprendizaje donde los valores de la responsabilidad, solidaridad, constancia, cooperación, y compromiso permiten que el estudiante colabore con el cuidado del medio ambiente a la vez que adquiere y produce nuevos conocimientos dando respuesta a los objetivos planteados en la agenda 2030.

La huerta escolar: espacio pedagógico para la implementación de una propuesta de integración curricular desde la formación agropecuaria" de la Institución Educativa Rural Agrícola del municipio de San Jerónimo. "Es un proyecto con dos líneas de investigación pedagógica y técnica, que permite desde la huerta escolar dinamizar los procesos de enseñanza aprendizaje, se crea una malla curricular desde las diferentes áreas del conocimiento de manera transversal que permite enseñar, crear y compartir saberes en los diferentes miembros de la comunidad educativa se realza el valor del trabajo colaborativo. (Méndez, A., Jiménez, 2016).

El proyecto de investigación "Hacia una praxis ecológica desde la huerta escolar, un estudio desde la pedagogía", "utiliza una metodología cualitativa donde a través de la cartografía social y la caja de arena se desarrolla un aprendizaje interdisciplinario que permite mejorar el aprendizaje desde la pedagogía de Waldorf donde los ambientes libres y de tipo colaborativo permiten mejores procesos del conocimiento". (Bolaños, J., Cifuentes, Figueroa, 2017) le da la relevancia a la transversalidad en las diferentes áreas del conocimiento, donde se presenta la huerta escolar como el ambiente fuera del aula de clase para que el estudiante aprenda nuevos conocimientos desde la practica utilizando el entorno con el que convive, el papel del docente acá es fundamental para que la transversalidad se haga de manera concreta es decir , "comparte la definición de la ciencia como construcción social y del conocimiento como herramienta de interpretación de la realidad ligado a la práctica social en que se genera" (Velásquez , 2009)

La experiencia significativa: ¡Qué divertido es aprender las matemáticas cultivando la tierra!, "este proyecto aprovecha los recursos de la región y tranversalizan las matemáticas con las actividades agrícolas de la región siembra, la cosecha y la recolección, utilizan medidas ancestrales y actuales para investigar el costo de los alimentos, es decir en este se desarrolla de manera específica el pensamiento numérico". (Álvarez, Domicó, 2016), ofrece a la investigación la importancia de utilizar "adecuadas prácticas sobre la siembra y la cosecha, que permitan potenciar el amor por las matemáticas, generar hábitos de buena salud y así, contribuir con la transformación social, mejorando el aspecto académico, la alimentación y prevención de enfermedades" ya que no solo era aprender números también era fundamental valorar el espacio rural que poseen y sacarle provecho desde su finca y sin duda el trabajo familiar les permitió un trabajo en conjunto.

El huerto como laboratorio de matemáticas: aprendizaje de los números racionales positivos, (Tesis de investigación presentada como requisito parcial para optar al título de: Magíster en Enseñanza de las Ciencias Exactas y Naturales) de la Universidad Nacional de Colombia confirma lo planteado en este proyecto cuando expresa "Son múltiples las ocasiones en las que tendrán que utilizar y relacionar los números y las distintas 
operaciones y formas de expresión y razonamiento matemático, en las tareas incluidas en el huerto escolar. Por ejemplo: plano del terreno, cálculo de superficies, áreas y volúmenes, mediciones diversas; altura de plantas, diámetros; medidas, estimación y cálculos de magnitudes, organización de la información, gráficas y estadísticas." (Cuenca, 2014). Esto permite que desde el contexto adquieran un aprendizaje significativo "es el mecanismo humano, por excelencia, para adquirir y almacenar la inmensa cantidad de ideas e informaciones representadas en cualquier campo de conocimiento". (Ausubel, 1963)

El huerto, un aula transversal, Grupo ecológico el germinado, Blog Agricultura Social, Lavapiés, Madrid, España. Es un semillero donde trabajan desde las huertas escolares de una manera transversal que permite acciones participativas, democráticas y sobre todo vivenciales que rompen con la manera tradicional de enseñanza. Así mismo, permite evolucionar y transformar de manera continua el proceso enseñanza aprendizaje. (Torres, 2015), lo valioso e interesante de trabajar con la huerta escolar es que da espacios para realizar procesos de enseñanza aprendizaje desde la articulación de diferentes áreas, lo que permite ratificar el aprendizaje significativo pues la vivencia desde el contexto real le da más credibilidad al estudiante del conocimiento que está adquiriendo, el aprovechar cada espacio de la huerta es el pretexto perfecto para enseñar "cuando se cosechan legumbres se explican las operaciones básicas, repartos proporcionales, estimación de medidas, medición de áreas, estimación de número de semillas, conceptos ancestrales, estudio del plano del terreno, registro de hortalizas, peso, dimensiones, medida del crecimiento de las plantas y sus partes, control económico del huerto, presupuesto, gastos"(Álvarez, Domicó,2016) esto le da un sentido diferente a las matemáticas y no solo se ven como el desarrollo del pensamiento lógico de estas sino que se le da un funcionalidad distinta y es la matemática financiera donde el niño o niña en conjunto con sus padres y maestros logran palpar que tener una huerta no solo es el laboratorio para adquirir un aprendizaje sino que el tenerla les disminuye gastos o puede prestarse para formar en un futuro una microempresa.

En este estudio se observan el desarrollo de los pensamientos numérico, geométrico y variacional, los estudiantes interpretan gráficas estadísticas, y a la vez desarrollan la competencia argumentativa al crear nuevas tablas con ejercicios sencillos de aplicación al nivel del conocimiento que se requiere en el área de matemáticas.

Este proyecto es innovador ya que presenta el área de matemáticas de manera lúdica donde la transversalidad de las áreas es fundamental. Se logra impactar tanto a los estudiantes, como a los padres de familia y sin contar con un recurso tan importante como lo es el internet, la huerta escolar es la excusa perfecta para resolver problemas matemáticos desde los diferentes pensamientos del área de matemáticas.

Este proyecto permite al estudiante salir del aula de clase y convertir su entorno en el mejor laboratorio de aprendizaje, donde la huerta de su casa, le sirve de retroalimentación al aplicar en ella los conocimientos teóricos aprendidos en el aula de clase y convertirse en un agente activo de cambio de su comunidad en compañía de su familia y docente.

\section{Conclusiones}

Los estudiantes a través de la transversalización de las áreas del conocimiento adquieren de una manera más agradable los procesos académicos permitiéndoles de esta manera mejorar su rendimiento a nivel de las competencias matemáticas. El desarrollo del pensamiento geométrico es mucho más práctico pues los estudiantes al medir las huertas reales le encuentran una aplicabilidad y luego al hacerlo en el computador es mucho más sencillo. Se puede evidenciar que la resolución de problemas tanto de números naturales como fraccionarios se convierte en un juego pedagógico donde ellos mismos crean problemas de compra y venta que se aplican en su vida cotidiana. También la aplicación de software como hot potatoes o Cuadernia le da al estudiante nuevas metodologías o estrategias para llegar al conocimiento de una manera más creativa. Con relación a la huerta escolar han aprendido a comer de manera saludable reconociendo las vitaminas y minerales de las verduras.

La participación de los padres en el acompañamiento a sus hijos desarrolló el trabajo de corresponsabilidad entre padres de familia y la Institución Educativa. Finalmente, proyectos de investigación como este puedan renovar y resignificar el Proyecto Educativo Institucional para lograr de esta manera cambios en el entorno y en los mismos agentes que participan en ellos. 
La Institución Educativa formalmente recibió el proyecto a satisfacción, por su parte, el rector después de leerlo y analizarlo, manifestó que lo tomará como referencia para otras áreas.

\section{Referencias bibliográficas}

Ausubel, D, (1963) recuperado en https://www.academia.edu/11982374/TEOR\%

C38DA_DEL_APRENDIZJE_SIGNIFICATIVO_TEORIA_DEL_ APRENDIZAJE SIGNIFICATIVO

Álvarez, J., Domicó, E., (2016) ¡Qué divertido es aprender las matemáticas cultivando la tierra! Recuperado en http://funes.uniandes.edu.co/10301/1/\%C3\%81/varez2016Qu\%C3\%A9.pdf

Bolaños, J., Cifuentes, M., Figueroa, L. (2017) Hacia una praxis ecológica desde la huerta escolar recuperado en https://dialnet.unirioja.es/servlet/articulo?codigo=6157571 Cuenca, G., (2014) El huerto como laboratorio de matemáticas: Aprendizaje de los números racionales positivos recuperado en http://bdigital.unal.edu.co/12898/1/7811510.2014.pdf

Duque, I. (2016) La investigación como estrategia pedagógica en el aula de clase

Kilpatrick, Gómez y Rico (1995) Errores y dificultades de los estudiantes Resolución de problemas Evaluación Historia, recuperado en https://core.ac.uk/download/pdf/12341271.pdf MEN (2004), Estándares básicos en matemáticas, recuperado de http://www.mineducacion.gov.co/1621/ articles-116042 archivo pdf2.pdf

Méndez, A. Jiménez, E. (2016). La huerta escolar: espacio pedagógico para la implementación de una propuesta de integración curricular desde la formación agropecuaria en la Institución Educativa Rural Agrícola del municipio de San Jerónimo recuperado en repository.upb.edu.co/bitstream/ handle/20.500.11912/2590/Trabajo\%20de\%20Grado\%20Natalia\%20Méndez\%20y\%20Edison\%20 Jiménez.pdf?sequence=1

Reyes, O. (2013). Inteligencias Múltiples y Desarrollo de Habilidades Investigativas de estudiantes de Postgrado en Línea. Memorias del Congreso UNED-ICDE. España

Sánchez, F. (2016). Los alumnos deben pasar de ser consumidores a creadores de conocimiento, recuperado en http://otrasvoceseneducacion.org/archivos/187745

Segovia, I. (2010) El reto es consolidar el sistema de calidad educativa, recuperado en https://www. mineducacion.gov.co/1621/article-242097.html

Suárez, L. (2015) Resolviendo problemas matemáticos desde la huerta escolar. Recuperado en http:/ aprendiendodesdelahuertaescolar.blogspot.com/

Torres, E (2015) El huerto, un aula transversal, Grupo ecológico el germinado, Blog

Agricultura Social recuperado en www.agriculturasocial.org/el-huerto-un-aula-transversal/

Velásquez, J. (2009) La transversalidad como posibilidad curricular desde la educación ambiental, https://

nedalyc.org/pdf/1341/134116861003.pdf

Zubiría, J, (2006) Los modelos pedagógicos: Hacia una pedagogía dialogante, recuperado en https://books. google.com.co/books?id = NspEAAAQBAJ\&pg=PT117\&lpg=PT117\&dq 